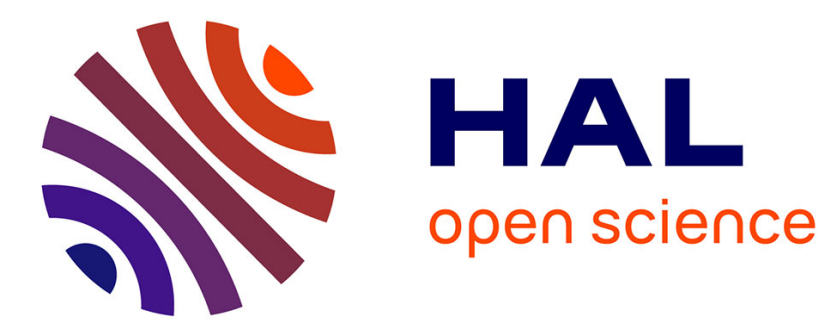

\title{
A stochastic subgrid model for sheared turbulence Jean-Pierre Bertoglio
}

\section{To cite this version:}

Jean-Pierre Bertoglio. A stochastic subgrid model for sheared turbulence. Lecture Notes in Physics, 1985, 230, pp.100-119. 10.1007/3-540-15644-5 . hal-00239481

\section{HAL Id: hal-00239481 \\ https://hal.science/hal-00239481}

Submitted on 5 Feb 2008

HAL is a multi-disciplinary open access archive for the deposit and dissemination of scientific research documents, whether they are published or not. The documents may come from teaching and research institutions in France or abroad, or from public or private research centers.
L'archive ouverte pluridisciplinaire HAL, est destinée au dépôt et à la diffusion de documents scientifiques de niveau recherche, publiés ou non, émanant des établissements d'enseignement et de recherche français ou étrangers, des laboratoires publics ou privés. 
J.P. Bertoglio

Laboratoire de Mécanique des Fluides

Ecole Centrale de Lyon

36 Avenue Guy de Collongue

69131 Ecully Cedex - France.

\section{Abstract}

A new subgrid model for homogeneous turbulence is proposed. The model is used in a method of large Eddy simulation coupled with an E.D.Q.N.M. prediction of the statistical properties of the small scales. The model is stochastic in order to allow a "desaveraging" of the informations provided by the E.D.Q.N.M. closure. It is basedon stochastic amplitude equations for two-point closures. It allows backflow of energy from the small scales, introduces stochasticity into I.E.S., and is well adapted to non isotropic fields. A few results are presented here.

\section{Introduction}

Direct Numerical Simulation is certainly today one of the major tools for the study and prediction of turbulence. However, it is known that, for flows at large Reynolds numbers it is not feasible to make a Euil Simulation over the whole range of scales of the turbulent spectrum. Only the large scales can be simulated, and the small eddies, or subgria scales, have to be modelled (see for exemple Ferziger, 1982). Erom a spectral point of view, it is possible to explicity take into account the turbulent motion up to a wavenumber cutoff $: \mathrm{Kc}$ and the terms representing the exchanges across $\mathrm{Kc}$ have to be parameterized.

Two-point closures provide an helpful analytical framework in which to investigate and developp subgrid models. They are believed to take correctly into account the exchange of energy between eddies of various sizes, accordingly, they are considered as valuable tools for the evaluation of energy flows across the wave number cutoff. Kraichnan (1976), Leslie et al. (1979) and chollet et al. (1981) have particularly used two-point theories to test and improve existing models in the caseof isotropic turbulence. 
In Kraichnan (1976), Leslie (1979) and Chollet (1981) the existence of a universal behaviour for the small scale spectrum was assumed, which implies that $\mathrm{K}_{\mathrm{C}}$ was supposed to be situated in the inertial range. It was then possible to derive subgrid models in which the parameterization was only made in terms of large scale quantities. Such models can therefore be used in L.E.S. without requiring an explicit computation of the small scale spectrum.

More recently, Chollet (1983) and Aupoix et al. (1983) have proposed method coupling L.E.S. and E.D.Q.N.M. This type of methods simultaneously involves a direct simulation of the large eddies and a twopoint closure computation of the small scales. At each time step, an information concerning the whole range of the spectrum is then available. Such methods are well adapted to predict either situations in which the small scales are not in equilibrium or situations in which the wave number cutoff is not in the inertial range. They can also take into account finite Reynolds number effects.

However an important problem is encountered when applying twopoint closures to subgrid models : the informations given by the closures are only statistically averaged informations, whereas one needs to account for the effect of small eddies on the particular realization of the field which is being simulated. The problem is known as the desaveraging problem (Basdevant et al. (1978)).

A simple way to solve the desaveraging problem is to introduce the concept of eddy viscosity. One of the advantages of subgrid models based on this concept is in particular that they ensure a drain of energy from the large scales to the small scales. In the case of isotropic three-dimensional turbulence eddy viscosity formulations were found to be consistent with the classical two-point closures (Kraichnan, 1976 ; Leslie, 1979 ; Chollet, 1981), and to lead to good predictions of the energy decay. The limitations on the use of eddy viscosity are however known. They have been pointed out by kraichnan (1976) in the case of two-dimensional isotropic turbulence. For threedimensional non isotropic turbulence we have shown, in an earlier study (Bertoglio and Mathieu, 1983), that the representation by an eddy viscosity was only justified for one part of the transfer.

Two deficiencies of eddy viscosity have in particular been pointed out : first, it does not provide for the possibility of back-flow of energy from the small scales, secondly it is not stochastic and 
does not account for the random forcing of small scales on large scales (Rose, 1977). In the case of a non isotropic turbulence subjected to a uniform mean shear flow, the importance of the first deficiency was found to be particularly large (see Bertoglio \& Mathieu, 1983). As for the lack of stochasticity, its consequences appear when the problem of predictability is considered. Let us for example consider two turbulent fields, which are initially supposed to have identical large scales and to differ only in their small scales. If the initial "error" is situated in the subgrid range of the spectrum, the eddy viscosity will never allow the "error" to contaminate the large eddies : the large scales of the two fields will always remain identical. This is known not to be a correct prediction.

In this paper, we present a new subgrid model in which the "desaveraging" operation is made by introducing a stochastic term. The model allows back-flow of energy from the small scales. It is based on the stochastic models for the analytical theories of turbulence (Kraichnan, 1961, 1971 ; Leith, 1971 ; Frisch et al., 1974). The cases of stochastic terms with and without memory are investigated.

The model is used in a "coupled" method in which the small scale spectrum is taken into account by the Eddy Damped Quasi Normal closure (Orszag, 1970), and the large scales are simulated by using a spectral algorithm. Results are first given in the case of isotropic turbulence. Extensions to the study of predictability and non isotropic sheared turbulence are shortly mentioned and a few results are presented.

\section{Stochastic models for two-point closures}

First introduced by Kraichnan (1961), stochastic models are equations for the turbulent fluctuation which are different from the original Navier-stokes equations in a sense that the original non linear term has been altered in a random fashion. They are helpful tools for turbulence theory since they share with the original equations many interesting features. A remarkable property of stochastic models is that they lead to a closed set of equations for averaged quantities, such as double velocity correlations, without having to introduce further assumptions.

In particular, a stochastic model leading to the equations of the 
D.I.A. was proposed by Kraichnan (1970), and Leith (1971) presented a stochastic equation corresponding to the E.D.Q.N.M. closure. We recall here both models. In the case of D.I.A. the amplitude equation for an isotropic turbulent field in which we include a solenoidal stirring force $F_{i}$ in order to permit stastical stationarity, is :

$\left\{\frac{\partial}{\partial t}+v K^{2}\right\} u_{i}(\vec{K}, t)+\int_{0}^{t} \sigma(K, t, s) u_{i}(\vec{K}, s) d s=q_{i}(\vec{K}, t)+f_{i}(\vec{K}, t)(1)$ where

$$
\sigma(K, t, s)=\pi K \iint_{\Delta} b_{K P Q} G(P, t, s) U(Q, t, s) P Q d P d Q
$$
is a quantity characterizing a damping and $q_{i}(\vec{K}, t)$ is a stochastic force given by :

$$
q_{i}(\vec{K}, t)=-i P_{i j m}(\vec{K}) \sum_{\vec{P}+\vec{Q}=\vec{K}} \xi_{j}(\vec{P}, t) \xi_{m}^{\prime}(\vec{Q}, t)
$$

where $\xi$ and $\xi^{\prime}$ are stochastic variables statistically independent of each other and such that:

$$
\left\langle\xi_{i}(\vec{K}, t) \xi_{j}\left(\vec{R}, t^{\prime}\right)\right\rangle=\left\langle\xi_{i}^{\prime}(\vec{K}, t) \xi_{j}^{\prime}\left(\vec{R}, t^{\prime}\right)\right\rangle=\left\langle u_{i}(\vec{K}, t) u_{j}\left(\vec{R}, t^{\prime}\right)\right\rangle,
$$

$G(P, t, \Delta)$ is the average infinitesimal response fonction, $U\left(K, t, t^{\prime}\right)$ is the modal time covariance :

$$
\left(\delta_{i j}-\frac{K_{i} K_{j}}{K^{2}}\right) \frac{U(K, t, s)}{2}=\lim _{L \rightarrow \infty}\left(\frac{L}{2 \pi}\right)^{3}\left\langle u_{i}(\vec{K}, t) u_{j}(-\vec{K}, t)\right\rangle,
$$

the integration $\Delta$ is over all $P$ and $Q$ such that $K, P, Q$ form a triangle, $P_{i j m}(\vec{k})$ satisfies :

$$
P_{i j m}(\vec{K})=\frac{1}{2}\left\{K_{l}\left(\delta_{i j}-\frac{K_{i} K_{i}}{K^{2}}\right)+K_{j}\left(\delta_{i l}-\frac{K_{i} K_{l}}{K^{2}}\right)\right\}
$$

and $b_{K P Q}$ is a coefficient depending on the geometry of the triad:

$$
b_{K P Q}(P / K)\left(x y+z^{3}\right)
$$


$x, y, z$ are the interior-angle cosines opposite $K, P, Q$, respectvery. $L$ is the side of a cyclic box.

The model proposed by Leith for E.D.Q.N.M. can be considered as a degenerated form of (1), in which all the quantities are evaluated at the same time, and $q_{i}$ is a white-noise process :

$$
\left\{\frac{\partial}{\partial t}+v K^{2}+\sigma(K, t)\right\} u_{i}(\vec{K}, t)=q_{i}(\vec{K}, t)+f_{i}(\vec{K}, t)
$$

where

$$
\sigma(K, t)=\pi K \iint_{\Delta} b_{K P Q} \theta_{K P Q}(t) U(Q, t) P Q d P d Q
$$

and

$$
q_{i}(\vec{K}, t)=-i P_{i j m}(\vec{K}) \sum_{\vec{P}+\vec{Q} \vec{K}} w(t)\left[\theta_{K P Q}(t)\right]^{1 / 2} \xi_{j}(\vec{P}, t) \xi_{m}^{\prime}(\vec{Q}, t)
$$

in which $w$ is a white noise process such that :

$$
\left\langle w(t) w\left(t^{\prime}\right)\right\rangle=2 \delta\left(t-t^{\prime}\right)
$$

It is worth noting that in the E.D.Q.N.M. formulation, the memory effects originally appearing in the D.I.A. equations have been antificially replaced by introducing a characteristic time $\theta_{K P Q}(t)$. We shall use here the expression (Bouquet et al. 1975) :

$$
\theta_{K P Q}(t)=\frac{1-e^{-[\eta(K, t)+\eta(P, t)+\eta(Q, t)] t}}{\eta(K, t)+\eta(P, t)+\eta(Q, t)}
$$

where

$$
\left.\eta(K, t)=.355 \iint_{0}^{k} P^{2} E(P, t) d P\right\}^{1 / 2}+0 K^{2}
$$




\section{Application to subgrid models}

As pointed by Kraichnan (1970), these stochastic model equations are a logical starting-point for using two-point closures for deriving subgrid scale models. A straighforward way to derive such a model would be to simply take into account the non linear interactions involving at least one wavenumber larger than $K_{c}$ by a stochastic amplitude equation, meanwhile interactions involving only wavenumbers smaller than $K_{c}$ would be explicitely computed in the original Navier stokes formulation. In the case of E.D.Q.N.M., this would lead to:

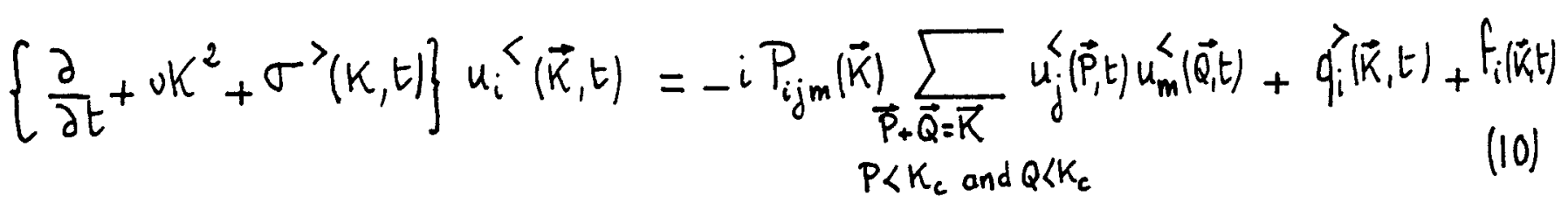

for the explicit scales $u_{i}^{<}\left(K<K_{c}\right)$, and :

$\left.\left\{\frac{\partial}{\partial t}+v k^{2}+\sigma(k, t)\right\} u_{i}\right\rangle(\vec{k}, t)=q_{i}(\vec{k}, t)+F_{i}(\vec{k}, t)$

for the subgrid modes $u_{i}^{>}\left(K>K_{c}\right)$, where $\sigma$ and $q_{i}$ are respectively given by (6) and (7), where:

$$
q_{i}^{>}(\vec{K}, t)=-i P_{i j m}(\vec{K}) \sum_{\substack{P>K_{c} \text { and } / o r \\ P}>K_{c}} w(t)\left[\theta_{K P Q}(t)\right]^{1 / 2} \xi_{/ j}(\vec{P}, t) \xi_{m}^{\prime}(\vec{Q}, t)
$$

and where :

$$
\sigma^{>}(K, t)=\pi K \iint_{\Delta^{\prime}} b_{K P Q} \theta_{K P Q}(t) U(Q, t) P Q d P d Q
$$

the integration being all over the part of the $P, Q$ plane-where $P>K_{c}$ and/or $Q>K_{c}$ and where $K, P, Q$ can form a triangle. $U(Q, t)$ is defined by :

$$
U(Q, t)=U(Q, t, t)
$$

We can at this point remark that the only informations concerning the subgrid scales in the supergrid equation (10) are statistically averaged, therefore equation (11) can be replaced by the master equation for the kinetic energy spectrum $E(K, t)$ :

$$
\left\{\frac{\partial}{\partial t}+20 K^{2}\right\} E(K, t)=t_{r}(K, t)
$$

in which tr is the usual E.D.Q.N.M. transfer term. 
Unfortunately the complete evaluation of $q_{i}^{>}$would require to much computational effort. Summing over all the subgrid-modes would result in a task comparable with the one required for a Full Direct Simulation. In the case of a D.I.A. type model, storage problems would furthermore be introduced by the explicit presence of memory effects.

our prescription is to replace $q_{i}(\vec{k}, t)$, which is a sum of products of stochastic processes, by a single stochastic process wich would satisfy relation (12) only in a statistically averaged way. If we name

$T_{i}{ }^{+}(\vec{k}, t)$ this new stochastic process, the equation for the explicit scales (10) then becomes:

$$
\begin{aligned}
\left.\left\{\frac{\partial}{\partial t}+u K^{2}+\sigma\right\rangle(K, t)\right\} u_{i}^{\langle}(\vec{K}, t)= & -i P_{i j m}(\vec{k}) \sum_{\substack{\vec{P}+\vec{Q}=\vec{K} \\
P<K_{c} \text { and } Q<K_{c}}} u_{j}^{<}(\vec{P}, t) u_{m}^{<}(\vec{Q}, t) \\
& +T_{i}^{+}(\vec{K}, t)+f_{i}(\vec{K}, t) \sum_{\left(K<K_{c}\right)}^{(15)}
\end{aligned}
$$

and the condition on $\mathrm{T}_{i}^{+}$can be written as :

$$
\left\langle T_{i}^{+}(\vec{K}, t) T_{j}^{+}\left(\vec{R}, t^{\prime}\right)\right\rangle=\left\langle q_{i}^{>}(\vec{K}, t) \quad q_{j}^{>}\left(\vec{R}, t^{\prime}\right)\right\rangle
$$
lations :

Using the definition of $q_{i}^{>}$, we can evaluate the two time corre-

$$
\begin{array}{rlrl}
\left.\left.\left.\left\langle q_{i}\right\rangle \vec{K}, t\right) q_{j}\right\rangle\left(\vec{R}, t^{\prime}\right)+q_{j}^{?}(\vec{R}, t) q_{i}^{\rangle} \mid \vec{K}, t^{\prime}\right) & =\lim _{L \rightarrow \infty}\left(\frac{2 \pi}{L}\right)^{3} \delta\left(t-t^{\prime}\right)\left(\delta_{i j}-\frac{K_{i} K_{j}}{K^{2}}\right) \frac{\left.t_{r}^{+}\right\rangle}{4 \pi K^{2}} & & \text { for } \vec{K}=-\vec{R} \\
& =0 & \text { for } \vec{K} \neq-\vec{R}
\end{array}
$$

where the quantity $t_{r}^{+}$, often called backscatter or input term, is given by :

$$
t_{r}{ }^{+>}(K, t)=\iint_{\Delta^{\prime}} \theta_{K P Q}(t) \quad b_{K P Q} \frac{K^{3}}{P Q} E(P, t) E(Q, t) d P d Q
$$

Replacing in equation (16), we obtain:

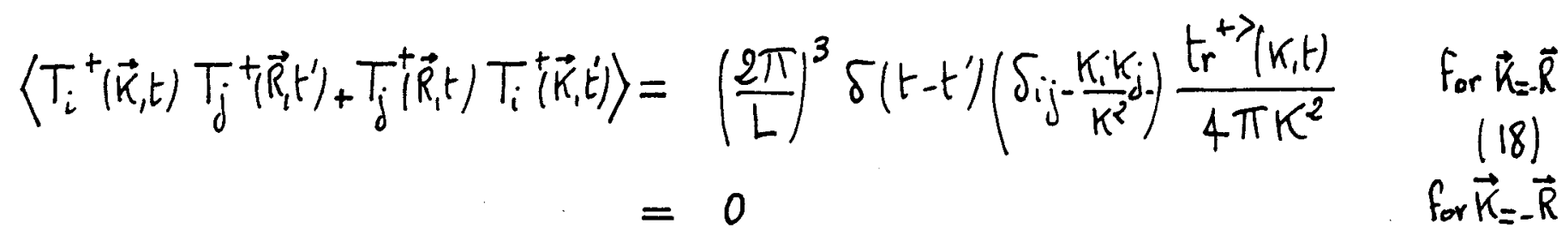

in which $L$ is assumed to be large. 
We have to specify now the statistical distribution of $T_{i}^{+} \cdot q_{i}{ }^{>}$ being a sum of a large number of stochastic terms, it seems reasonable to assume $T_{i}{ }^{+}$to be gaussian. We shall discuss how to generate $T_{i}^{+}$ in appendix 1 .

The final set of equations is then (13), (14), (15), (17) and (18), together with the relations characterizing the stochastic process (Al.1) and (A1.2). Equation (15) is the equation governing the evolution of the large scales, (14) is the rate equation for the small scale spectrum, and relations (13), (17) and (18) are characterizing the coupling terms: they take into account interactions across the wavenumber cutoff.

The operator< >appearing in (18), as well as in the definition of $U$ for $K\left\langle K_{c}\right.$ :

$$
\left(\delta_{i j}-\frac{K_{i} K_{j}}{K^{2}}\right) \frac{U(K, t)}{2}=\left(\frac{L}{2 \pi}\right)^{3}\left\langle u_{i}\left\langle(\vec{K}, t) u_{j}<(-\vec{K}, t)\right\rangle,\right.
$$

denotes ensemble average. In the case of L.E.S. we would have to consider $\mathrm{N}$ realizations, corresponding to $\mathrm{N}$ different initial conditions and $N$ different sets of random numbers in the generation of $T_{i}^{+}$, and average over the results. On the basis of practical considerations, it was set $N$ equal to 1 .

An advantage of the model immediately appears : it will introduce stochasticity in L.E.S. as we shall see in section 5 when the model will be applied to the study of predictability. A second advantage appears when anisotropic turbulence is considered. The equivalent of equation (18) can then be written as :

$$
\begin{aligned}
\left\langle T_{i}+(\vec{K}, t) T_{j}^{+}\left(\vec{R}, t^{\prime}\right)+T_{j}+\left(\vec{R}, t^{\prime}\right) T_{i}^{+}\left(\vec{K}, t^{\prime}\right)\right\rangle & =\left(\frac{2 \pi}{L}\right)^{3} \delta\left(t-t^{\prime}\right) T_{i j}^{+>}(\vec{K}, t) & \begin{aligned}
\text { for } \vec{K}=-\vec{R} \\
(1 g)
\end{aligned} \\
& =0 & \text { for } \vec{K} \neq-\vec{R}
\end{aligned}
$$

in which $T_{i j}^{+>}$is the anisotropic backscatter (for a complete expression of $T_{i j}^{+>}$see Bertoglio \& Mathieu, 1983). Since it is possible to find $T_{i}^{+}$satisfying (19), it then becomes possible to preserve completely the anisotropic characteristics of $T_{i j}+>$ in the subgrid model. This was impossible when using the classical concept of eddy viscosity as we pointed out in an earlies paper (Bertoglio \& Mathieu, 1983). we shall shortly discuss the application of the model to non isotropic sheared turbulence in section 5 . 
4. Introduction of a stochastic term with memory

The presence of a white-noise process in $T_{i}^{+}$can be judged to be rather unphysical, and one could try to improve the subgrid model presented in section 3 by introducing a memory effect in the stochastic process. Since memory is present in the model amplitude equation for D.I.A., relation (3) constitutes a logical starting point to make such an improvement.

It is however not our purpose here to work completely in the framework of D.I.A., computation of the small scales spectrum would be to cumbersome to permit extensions to anisotropic turbulence. Besides D.I.A. is not statistically Galilean Invariant.

Let us make crude assumptions and propose the following approach. We assume that the memory in $\mathrm{T}_{i}^{+}$is an exponentially decreasing fundtion of time :

$$
\begin{gathered}
\left\langle T_{i}^{+}(\vec{k}, t) T_{j}^{+}\left(\vec{k}, t^{\prime}\right)\right\rangle=\left\langle T_{i}^{+}(\vec{k}, t) T_{j}^{+}(-\vec{k}, t)\right\rangle e^{-\frac{t-t^{\prime}}{t_{N L}(k)}} \\
\left(t \geqslant, t^{\prime}\right)
\end{gathered}
$$

in which $t_{N L}(K)$ is a characteristic time.

It is then possible to determine $t_{N L}(K)$ by using the D.I.A. wtochastic model equation. This can be done in the case of a stationary turbulence, if we assume that the two-time velocity correlations are exponentially decreasing functions of time :

$$
\begin{gathered}
\left\langle u_{i}(\vec{K}, t) u_{j}\left(-\vec{K}, t^{\prime}\right)\right\rangle=\left\langle u_{i}(\vec{K}, t) u_{j}(-\vec{K}, t)\right\rangle e^{-\eta(k)\left(t-t^{\prime}\right)} \\
\left(t \geqslant t^{\prime}\right)
\end{gathered}
$$

The calculation is presented in appendix 2 .

The determination of the one time correlation $\left\langle T_{i}^{+}(\vec{K}, t) T_{j}^{+}(-\vec{K}, t)\right\rangle$ is some what more complex that in the case of the white-noise process, since E.D.Q.N.M. cannot now directly provide this quantity. Relation (18) was valid only for a white-noise. We have here to satisfy :

$$
\begin{aligned}
&\left\langle T_{i}^{+}(\vec{K}, t) u_{j}\left\langle(\vec{K}, t)+T_{j}^{+}(-\vec{K}, t) u_{i}^{\langle}\langle\vec{K}, t)\right\rangle=\right.\left(\frac{2 \pi}{L}\right)^{3}\left(\delta_{i j}-\frac{K_{i} K_{j}}{K^{2}}\right) \frac{\left.t_{r}{ }^{+}\right\rangle}{\left.4 \pi K^{2}, t\right)} \\
&\left(K<K_{c}\right)
\end{aligned}
$$




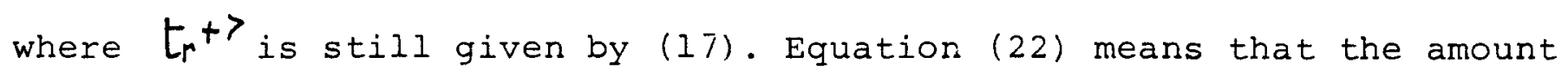
of energy injected in the super-grid modes by the stochastic process must be statistically equal to its E.D.Q.N.M. determination.

Since the velocity fluctuation appears in it, equation (22) cannot be used in a straightforward way. We have to write the left hand side in the form

$$
\begin{aligned}
\left\langle T_{i}^{+}(\vec{K}, t) u_{j}^{\prime}(-\vec{K}, t)\right. & +T_{j}^{+}(-\vec{K}, t) u_{i}\langle(\vec{K}, t)\rangle=\int_{0}^{t} G\left(\vec{K}, t, t^{\prime}\right) \\
& \times\left\langle T_{i}^{+}(\vec{K}, t) T_{j}^{+}\left(-\vec{K}, t^{\prime}\right)+T_{j}^{+}(-\vec{K}, t) T_{i}^{+}\left(\vec{K}, t^{\prime}\right)\right\rangle d t^{\prime}
\end{aligned}
$$

in which the infinitesimal response function was assumed to be statistically independent of $T_{i}^{+}$.

Assuming furthermore that :

$$
\begin{aligned}
G\left(\vec{K}, t, t^{\prime}\right)= & e^{-\frac{t-t^{\prime}}{\theta_{G}(K)}} \\
& \left(K \leqslant K_{c}, t \geqslant t^{\prime}\right)
\end{aligned}
$$

and using (20) and (23) give for a stationary turbulence:

$$
\begin{aligned}
2\left\langle T_{i}+(\vec{K}, t) T_{j}+(-\vec{K}, t)\right\rangle= & \\
& \left(\frac{2 \pi}{L}\right)^{3}\left[\frac{1}{\theta_{G}(K)}+\frac{1}{t_{N L}(K)}\right]\left(\delta_{i j}-\frac{K_{i} K_{j}}{K^{2}}\right) \frac{t_{r}{ }^{+>}\left(K_{1} t\right)}{4 \pi K^{2}}
\end{aligned}
$$

$\theta_{G}(K)$ is a characteristic time of the response of the large scale field, it can therefore be estimated at each time step from the result of L.E.S.

$t_{N L}(K)$ is the correlation time of the stochastic process. It can be determined by using (A23). It could also be arbitrarily fixed and used as the only adjustable parameter in the model. In the limit $t_{N L} \longrightarrow 0$ equations (25) and (20) degenerate into equation (18), which means that the memory model degenerated into the white-noise model. 
The damping term appearing in (15)

$$
\sigma>(k, t) \quad u_{i}<(\vec{k}, t)
$$

is not modified here. We do not use a term involving a time integration such as the one encountered in (1) on the basis of practical considerations.

The final set of equations for the model with memory is then : $(13),(14),(15),(17),(20),(25),(A I .1)$ and (AI.2), together with (A2.3) which specifies the correlation time of the subgrid term.

\section{Results}

The model has first been used to compute the decay of an isotropic turbulence. A numerical code has been written for the simulation of the large eddies, following the method proposed by orszag \& Patterson (1972). We used a $16^{3}$ grid. A few runs were performed with a $32^{3}$ grid. The initial data are generated as suggested by Rogallo (1981). Since the triple velocity correlations are initially equal to zero, we used relation (8) to specify the E.D.Q.N.M. characteristic time. This form ensures initial compatibility between L.E.S. and E.D.Q.N.M. since it also corresponds to $\langle u m u\rangle=0$ at $t=0$ (Aupoix, personnal communication).

The first results showed a discontinuity in the slope of the energy spectrum at the wavenumber cutoff. Our interpretation was that the effect of the damping term $\sigma\rangle$ on the characteristic correlation times of the supergrid modes was to strong to be compatible with E.D.Q.N.M... Transfers between supergrid modes were then underestimated in comparison with E.D.Q.N.M., and accordingly they were unable to balance the energy drain across the cutoff in a correct way.

This undesirable behaviour was cured by a modification of the E.D.Q.N.M. characteristic time for the supergrid modes. Our proposition is to identify $\eta(K, t)$ with the inverse of the characteristic time of the average response fonction, a quantity which is directly deduced from the computed scales:

$$
\eta(K, t)=\frac{1}{\theta_{G}(K, t)} \quad \text { for } K \leqslant K_{c}
$$


The damping term appearing in (15)

$$
\sigma>(k, t) \quad u_{i}<(\vec{k}, t)
$$

is not modified here. We do not use a term involving a time integration such as the one encountered in (1) on the basis of practical considerations.

The final set of equations for the model with memory is then : $(13),(14),(15),(17),(20),(25),(A I .1)$ and (AI.2), together with (A2.3) which specifies the correlation time of the subgrid term.

\section{Results}

The model has first been used to compute the decay of an isotropic turbulence. A numerical code has been written for the simulation of the large eddies, following the method proposed by orszag \& Patterson (1972). We used a $16^{3}$ grid. A few runs were performed with a $32^{3}$ grid. The initial data are generated as suggested by Rogallo (1981). Since the triple velocity correlations are initially equal to zero, we used relation (8) to specify the E.D.Q.N.M. characteristic time. This form ensures initial compatibility between L.E.S. and E.D.Q.N.M. since it also corresponds to $\langle u m u\rangle=0$ at $t=0$ (Aupoix, personnal communication).

The first results showed a discontinuity in the slope of the energy spectrum at the wavenumber cutoff. Our interpretation was that the effect of the damping term $\sigma\rangle$ on the characteristic correlation times of the supergrid modes was to strong to be compatible with E.D.Q.N.M... Transfers between supergrid modes were then underestimated in comparison with E.D.Q.N.M., and accordingly they were unable to balance the energy drain across the cutoff in a correct way.

This undesirable behaviour was cured by a modification of the E.D.Q.N.M. characteristic time for the supergrid modes. Our proposition is to identify $\eta(K, t)$ with the inverse of the characteristic time of the average response fonction, a quantity which is directly deduced from the computed scales:

$$
\eta(K, t)=\frac{1}{\theta_{G}(K, t)} \quad \text { for } K \leqslant K_{c}
$$


In order to ensure continuity of $\eta$ at $K=K_{c}$ we furthermore write :

$$
\eta(K, t)=\left[\frac{1}{\theta_{G}^{2}\left(K_{c}, t\right)}+[.355]^{2} \int_{K_{c}}^{K} p^{2} E(P, t) d P\right]^{1 / 2}+u K^{2}
$$

With this modification, the behaviour of the energy spectrum $E(K, t)$ appears to be correct. In figure 1 we have plotted a spectrum obtained in the case of a freely evolving isotropic turbulence. The $16^{3}$ grid has been used and the stochastic term includes memory effects ( $t_{N L}$ arbitrarily fixed).

On figure 2 the backscatter predicted by E.D.Q.N.M. $t_{r}{ }^{+>}(K, t)$ is compared with the effective value actually injected in L.E.S. by the stochastic term, called $t_{r}+{ }_{\text {eff }}$. The agreement is reasonably good. In this case the $32^{3}$ grid has been used. The stochastic process is without memory $\left(t_{N_{L}}=\Delta t\right)$. In the case of a stochastic process including memory effects, the agreement would be less satisfactory since, for a freely evolving turbulence, equation (25) is not exactly statisfied. On figure 2 , the term characterizing the drain of energy from the large scales to the small scales:

$$
t_{r}{ }^{->}(K, t)=2 \sigma(k, t) \quad E(K, t)
$$

has also been plotted.

Results concerning the turbulent kinetic energy decay are presented on figure 3. Evolutions of the turbulent kinetic energy are plotted. We compare the kinetic energy of the supergrid field to the kinetic energy of the full field. The stochastic process used here includes memory effects, the characteristic time $t_{N L}$ is given by (A2.3).

The advantage of including memory in $\mathrm{T}_{i}^{+}$does not appear clearly when comparing the results. Quantities like double velocity correlations, energy spectra, are only very slightly modified. Only the skewness of the large scale field seems to be affected.

It is beyond the scope of the present paper to give a full presentation of the extensions to the study of predictability and to the 


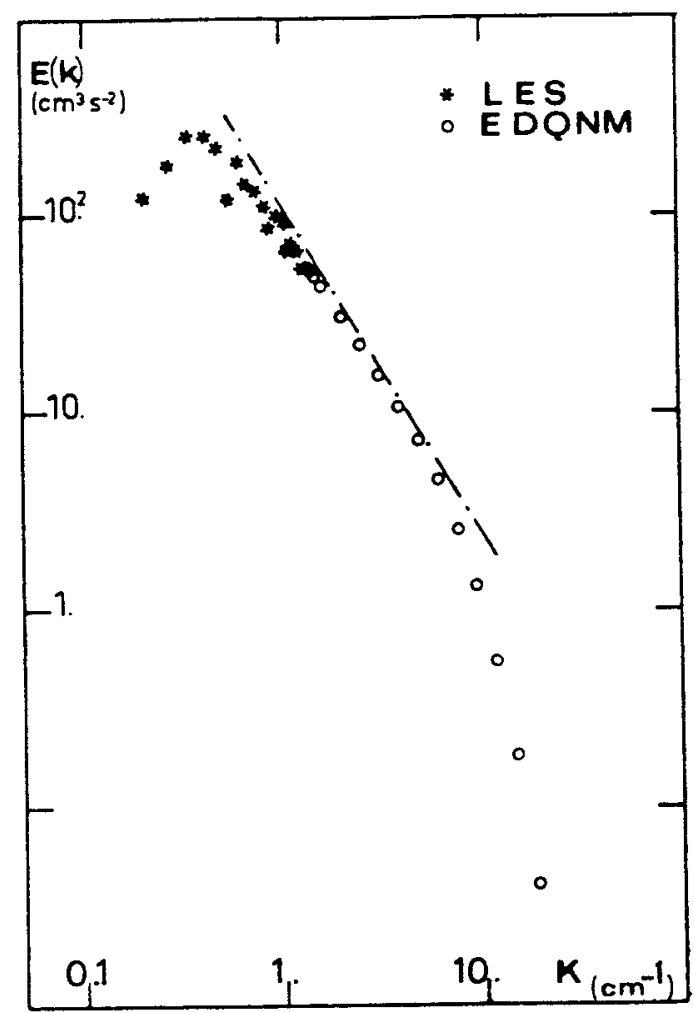

Figure I - Kinetic energy spectrum. Results of L.E.S. : *, and E.D.Q.N.M. $0 ; t=0.42 \mathrm{~s}, K_{c}=1.21 \mathrm{~cm}^{-1}, 16^{3} \mathrm{grid}, t_{N L}=0.15 \mathrm{~s}$.

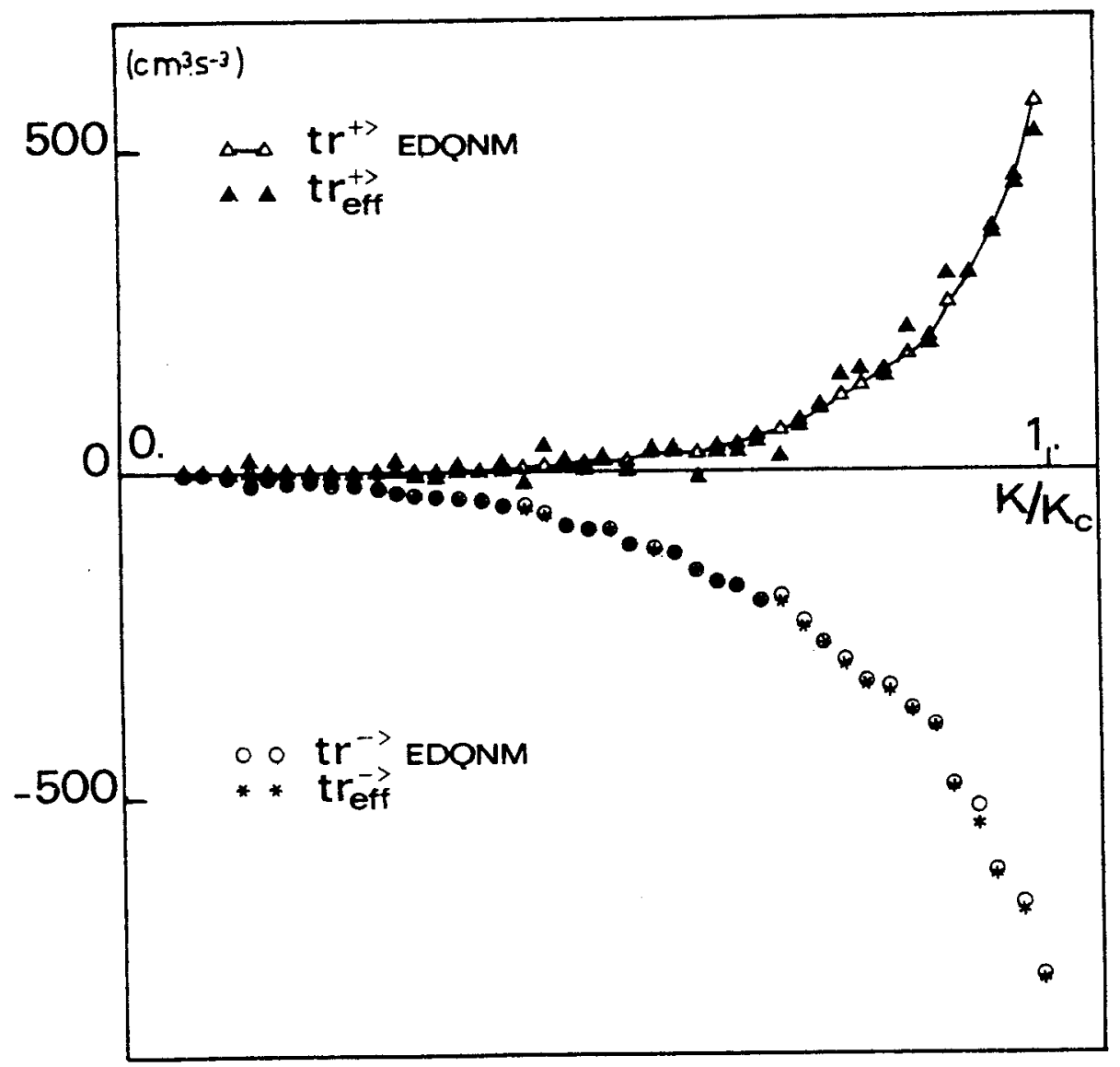

Figure 2 - Comparison between the E.D.Q.N.M. evaluation of the subgrid terms $t_{r}^{+}, t_{p}^{->}$, and the values effectively injected in the simulation: $t_{r_{\text {eff }}}+t_{r}$ eff $; t=0.5 \mathrm{~s}, 32^{3}$ grid, $t_{N L}=\Delta t$. 
prediction of non-isotropic homogeneous turbulence. Nevertheless some results are presented here as examples.

In the case of predictability two realizations of the large eddies have to be simulated, simulatneously with a closure computation of the subgrid energy spectrum and of the "error" spectrum. The correlation between the stochastic subgrid terms acting on the two realizations is fixed by the closure. On figure 4, the growth of the "error" appears in the spectra. On the first stage, only the subgrid eddies are contaminated. Latter the error affects the supergrid field. It is worth pointing out that the error spectrum $E_{\Delta}\left(K_{1} H_{\text {keeps }} K^{4}\right.$ slope on both sides of the cutoff.

When non-isotropic turbulence is investigated, a coordinate transformation have to be introduced in the spectral simulation (Rogallo, 1981). In the E.D.Q.N.M. computation, simplifications are introduced in order to reduce the computational cost (Bertoglio, 1981). The model is applied to an initially isotropic turbulence subjected to a uniform mean shear flow. Spectral results are plotted in figure 5, they are compared with results obtained with an eddy viscosity formulation, also coupled with E.D.Q.N.M.. The improvement due to the stochastic modelling of the backscatter appears on the component normal to the velocity in the plane of the shear $\varphi_{33}\left(K_{1}\right)$. Another interesting result is that the slope of the Reynolas stress spectrum tends to a $K^{-7 / 3}$ behaviour on both sides of $K_{c}$ (figure 6 ).

\section{Conclusion}

A new subgrid model has been presented. It has been shown to give satisfactory results in the case of isotropic turbulence. Since the model is stochastic it permits to take into account effects neglected by eddy viscosity formulations, for example in the case of predictability studies. When non isotropic turbulence is considered, the model seems to do better than eddy viscosity.

The model have been used in a method involving both L.E.S. and E.D.Q.N.M. computations. It is therefore quite uneasy to apply to industrial flows. It can however be used as a guidance to developp simpler model in which the stochastic modelling of energy back-flows could be retained. It can also be used to test "defiltering" methods (Bardina et al., 1983). 

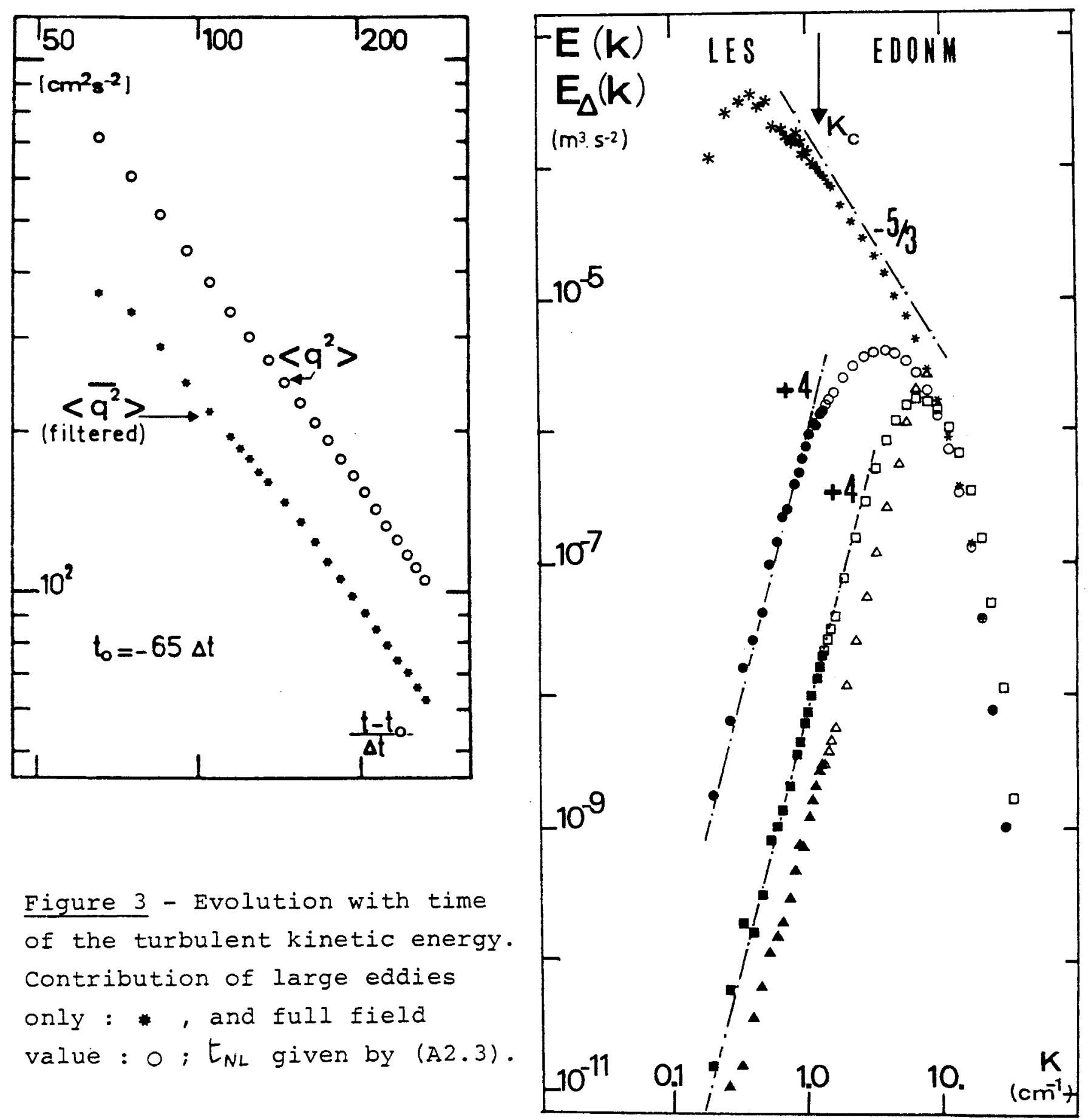

Figure 3 - Evolution with time of the turbulent kinetic energy. contribution of large eddies only : * and full field value : $0 ; t_{N L}$ given by (A2.3).

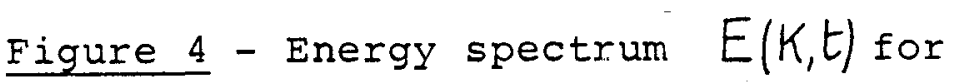
$t=0.1$ s, I.E.S. prediction : *, and E.D.Q.N.M. : * ; and error spectrum $E_{\Delta}(K, t)$ for $t=0.025 \mathrm{~s}$, L.E.S.: $\Delta$ and E.D.Q.N.M. : $\Delta ;$ for $t=0.05 \mathrm{~s}$, L.E.S. : and E.D.Q.N.M. : $\square$; and for $t=0.1$ S, L.E.S. and E.D.Q.N.M.: 


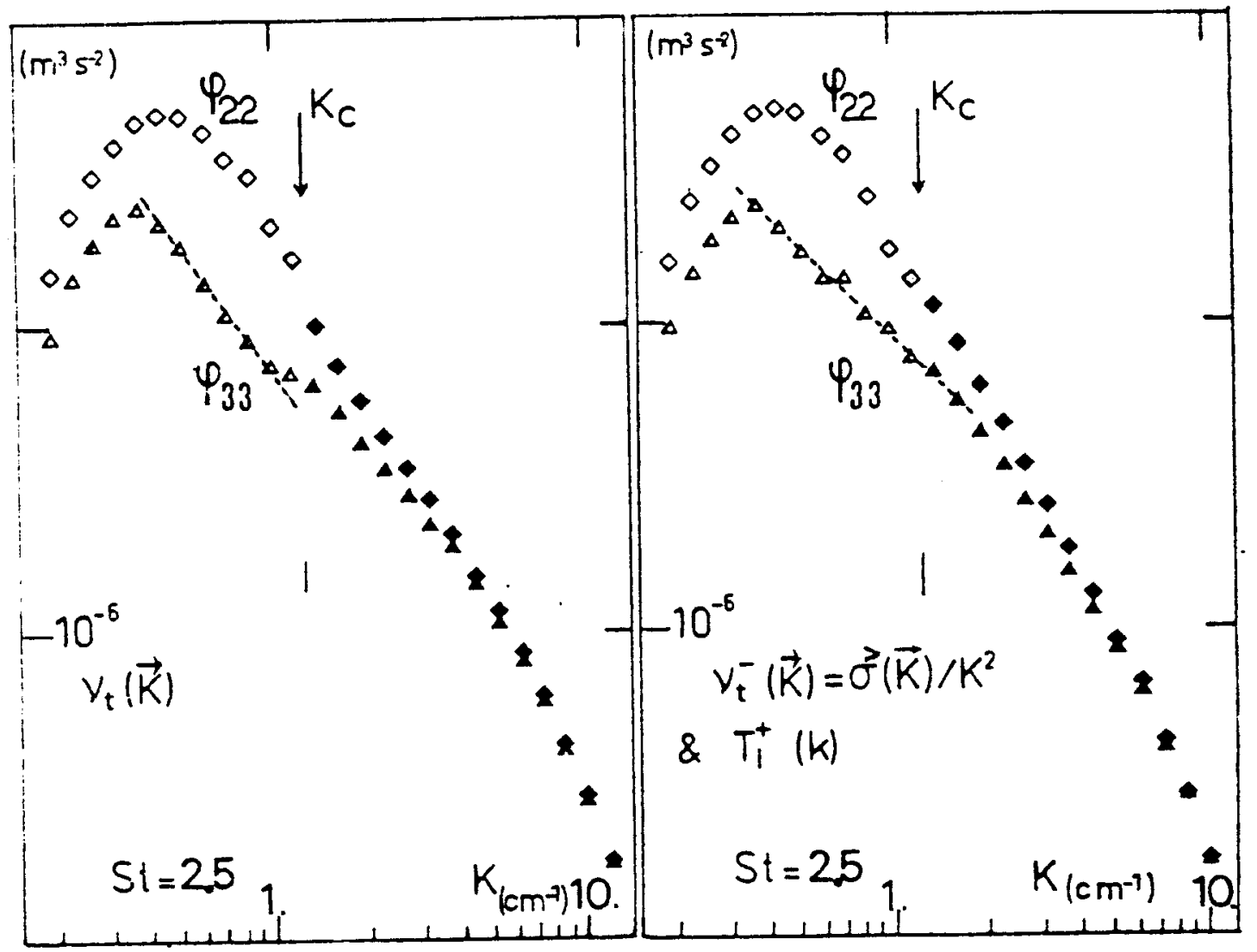

Figure 5 - Comparison between eddy viscosity and the present model. $\varphi_{22}$ and $\varphi_{33}$ are two components of the double correlation spectrum integrated over a spherical shell of radius $K$. The value of the shear is $S=12.5 \mathrm{~s}^{-1}$. The value of the shear is $S=12.5 \Delta$

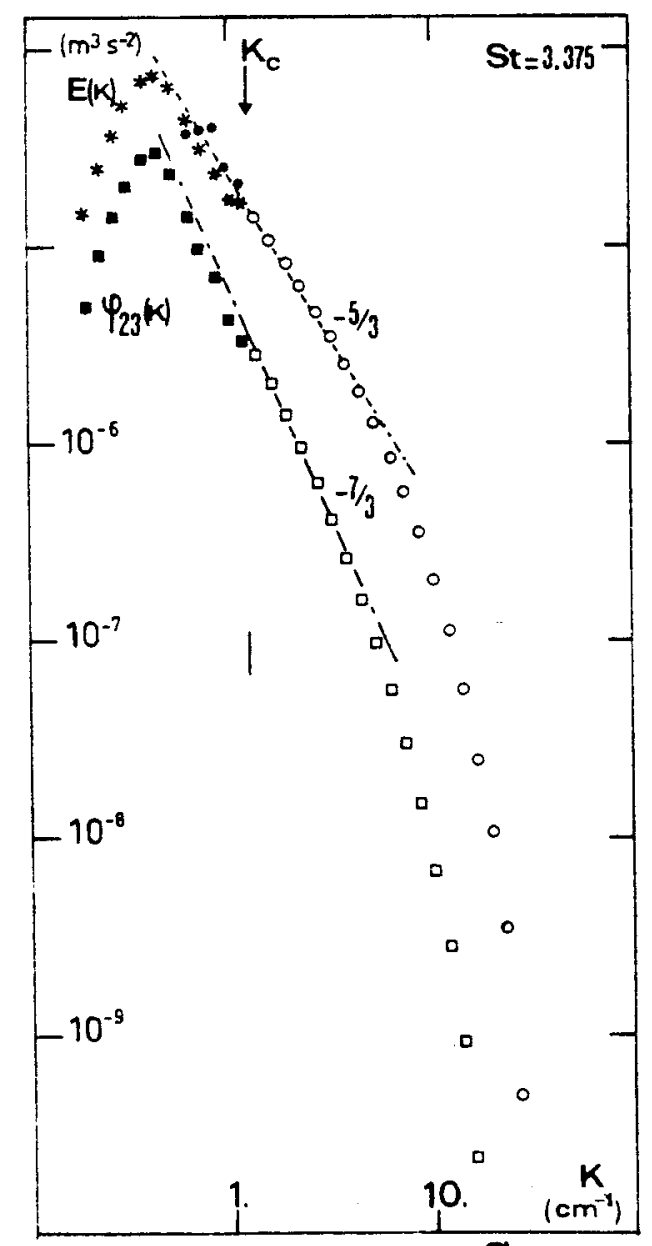

Figure 6 - Energy spectrum: $E(K, t)$,

L.E.S. : * and and E.D.Q.N.M.:O,

$\because \therefore$ Feylolds stress spectrum : $\varphi_{23}(K, t)$

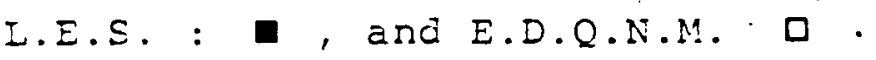
$S t=3.375$, in which $S$ is the value of the shear : $=12.5 \mathrm{~s}^{-1}$. 
Extensions to two-dimensional turbulence could be of interest since in this case back-flows of energy are important.

\section{Appendix 1 - Generation of a stochastic process}

We propose here a method to generate a stochastic terme such as the averaged quantity :

$$
\left\langle T_{i}^{+}(\vec{K}, t) T_{j}+\left(-\vec{K}, t^{\prime}\right)\right\rangle
$$

satisfiesrelation (18), or, more generally, relations (20) and (25). In other words, the stochastic process must have a given characteristic time and its single time correlation must be equal to a given value. we call this value $f_{i j}(\vec{K}, t)$.

Since incompressibility implies :

$$
K_{i} f_{i j}(\vec{K}, t)=0
$$

it is convenient to introduce a new frame, an axis of which is parallel to $\vec{K}$, for example $K_{3}$. In this new frame all components corresponding $i=3$ vanish. We can then consider that $i$ and $j$ take only the values 1 and 2 .

In this new frame $f_{i j}$ is an isotropic tensor if turbulence is isotropic. However, since the model is supposed to be valid for non isotropic fields, we consider the general case in which $f_{i j}$ is not isotropic. fij is real and symmetrical.

Details concerning the generation of are given in Bertoglio and Mathieu (1984). The final result is written in a discrete form and for a fixed value of $\vec{K}$. It is, at the time step number $(n)$ :

$$
\begin{aligned}
& T_{1}^{+}(n+1)=\left(1-\frac{\Delta t}{E_{N L}}\right) T_{1(n)}^{+}+\beta_{11(n+1)}\left(f_{11(n)} \frac{\Delta t}{t_{N L}}\right)^{1 / 2} e^{i 2 \pi \varphi_{(n+1)}} \\
&+\beta_{12(n+1)}\left(f_{22(n)} \frac{\Delta t}{E_{N L}}\right)^{i / 2} e^{i 2 \pi \varphi_{(n+1)}^{\prime}} \\
&(A 1.1 a)
\end{aligned}
$$




$$
\begin{aligned}
T_{2(n+1)}^{+}=\left(1-\frac{\Delta t}{E_{N L}}\right) T_{2(n)}^{+} & +\beta_{22(n+1)}\left(f_{22(n)} \frac{\Delta t}{E_{N L}}\right)^{1 / 2} e^{i 2 \pi \varphi_{2(n+1)}} \\
& +\beta_{12(n+1)}\left(f_{11 / n)} \frac{\Delta t}{E_{N L}}\right) e^{i 2 \pi \varphi_{(n+1)}^{\prime}}
\end{aligned}
$$

where $\Delta t$ is the time step, where the $\beta_{i j}$ are solutions of :

$$
\begin{aligned}
& \beta_{11(n+1)}^{2}=\frac{\left(f_{11(n+1)}-f_{11(n)}\right) t_{N L} / \Delta t-f_{22(n)} \beta_{12(n+1)}^{2}}{f_{11(n)}}+\left(2-\Delta t / t_{N L}\right) \\
& \beta_{22(n+1)}^{2}=\frac{\left(f_{22(n+1)}-f_{22(n)}\right) t_{N L} / \Delta t-f_{11(n)} \beta_{21(n+1)}^{2}}{f_{22(n)}}+\left(2-\Delta t / t_{N L}\right) \\
& \beta_{12(n+1)} \beta_{21(n+1)}=\frac{\left(f_{12(n+1)}-f_{12(n)}\right)^{t_{N L} / \Delta t}}{\left(f_{11(n)} f_{22(n)}\right)^{1 / 2}}+\frac{f_{12(n)}}{\left(f_{11(n)} f_{22(n)}\right)^{1 / 2}}\left(2-\Delta t / t_{N L}\right) \\
& \beta_{12(n+1)}^{2}=\beta_{21(n+1)}^{2} \\
& \varphi_{1(n)}, \varphi_{2(n)} \text { and } \varphi_{(n)}^{\prime} \text { constitute a set of random numbers independent }
\end{aligned}
$$

Appendix 2 - Determination of characteristic time $t_{N L}(K)$

starting from equation (20), and replacing $q_{i}{ }^{2}$ by its D.I.A. expression

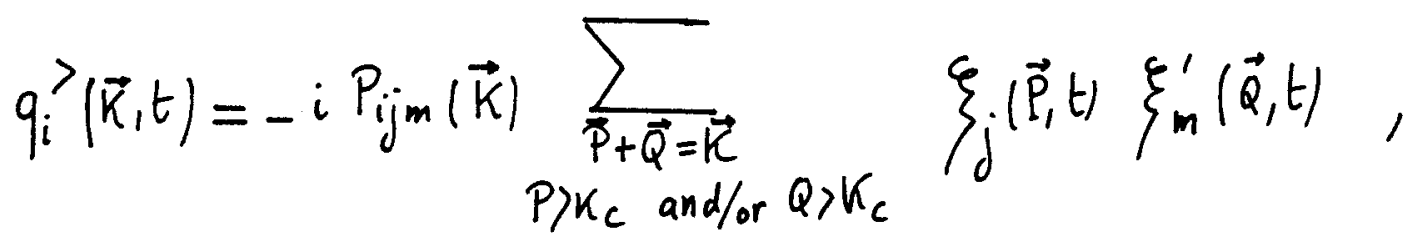

give : 


$$
\begin{aligned}
\left\langle T_{i}^{+}(\vec{K}, t) T_{j}^{+}\left(-\vec{K}, t^{\prime}\right)\right\rangle= & P_{i l m}(\vec{K}) P_{j n r}(\vec{K}) \sum_{P} \sum_{P+\vec{Q}=\vec{K}} \\
& \times\left(\left\langle u_{l}(\vec{P}, t) u_{n}\left(-\vec{P}, t^{\prime}\right)\right\rangle\left\langle u_{m}(\vec{Q}, t) u_{r}\left(-\vec{Q}, t^{\prime}\right)\right\rangle\right. \\
& \left.+\left\langle u_{l}(\vec{P}, t) u_{r}\left(-\vec{P}, t^{\prime}\right)\right\rangle\left\langle u_{m}(\vec{Q}, t) u_{n}\left(-\vec{Q}, t^{\prime}\right)\right\rangle\right)
\end{aligned}
$$

$(A 2-1)$

For a stationary turbulence, replacing the two time correlations by the exponential forms $(20)$ and (21), then integrating between $t^{\prime}=0$ and $t^{\prime}=t$ give:

$$
\begin{aligned}
& t_{N L}(K)\left\langle T_{i}^{+}(\vec{K}, t) T_{j}^{+}(-\vec{K}, t)\right\rangle=P_{i l m}(\vec{K}) P_{j n r}(\vec{K}) \\
& \sum_{\substack{\left.\vec{P}+\vec{Q}=\vec{K} \\
P\rangle K_{c} \text { and/or Q Q }\right) K_{c}}} \frac{1}{\eta(P, t)+\eta(Q, t)}\left\{\left\langleu_{l}(\vec{P}, t) u_{n}(-\vec{P}, t)\left\langle u_{m}(\vec{Q}, t) u_{r}(-\vec{Q}, t)\right\rangle\right.\right. \\
& \left.+\left\langle u_{\rho}(\vec{P}, t) u_{r}(-\vec{P}, t)\right\rangle\left\langle u_{m}(\vec{Q}, t) u_{n}(-\vec{Q}, t)\right\rangle\right\}
\end{aligned}
$$

for large values of $t$.

We can now use (A2.1) for $t=t^{\prime}$ to express $\left\langle T_{i}^{+}(\vec{K}, t) T_{j}^{+}(-\vec{K}, t)\right\rangle$ in (A2.2). We finally obtain that $t_{N L}$ is equal to the R.H.S. of (A2.2) divided by the R.H.S. of (A2.1). In the case of an isotropic turbulence it comes :

$$
t_{N L}(K)=\frac{\iint_{\Delta^{\prime}} \theta_{P Q}(t) b_{K P Q} \frac{K^{3}}{P Q} E(P, t) E(Q, t) d P d Q}{\iint_{\Delta^{\prime}} b_{K P Q} \frac{K^{3}}{P Q} E(P, t) E(Q, t) d P d Q}
$$

where :

$$
\theta_{P Q}(t)=\frac{1}{\eta(P, t)+\eta(Q, t)}
$$


Aupoix B., Cousteix J. and Liandrat J., 1983, Effects of rotation on isotropic turbulence. Fourth Int. Symp. Turb. Shear Flows, Karlsruhe.

Bardina J., Ferziger J.H. and Reynolds W.C., 1983, Improved turbulence models based on large eddy simulation of homogeneous, incompressible, turbulent flows. Stanf. Univ. Report NOTE-19, May 1983.

Basdevant D., Lesieur M. and Sadourny R., 1978, Subgrid-scale modeling of enstrophy transfer in two-dimensional turbulence. Journal of Atm. Sci., Vol. 35, pp. 1028-1042.

Bertoglio J.P., 1981, A model of three-dimensional transfer in Nonisotropic homogeneous turbulence. Third Int. Symp. Turb. Shear Flows, Davis, Sept. 81, Springer-Verlag, 1982.

Bertoglio J.P. and Mathieu J., 1983, Study of subgrid models for sheared turbulence. Fourth Symp. on Turb. Shear Flows, Karlsruhe, sept. 83.

Bertoglio J.P. and Mathieu J., 1984, Modélisation stochastique des petites échelles de la turbulence : génération d'un processus stochastique. C.R.Acad. Sci., to be published.

Chollet J.P. et Lesieur M., 1981, Parameterization of small scales of three-dimensional isotropic turbulence utilizing spectral closures. J. Atm. Sci., Vol. 38, pp. 2747-2757.

Chollet J.P., 1983, Two-point closure as a subgrid scale modeling for Large Eddy Simulations. Fourth Symp. on Turb. Shear Flows, Karlsruhe.

Frish U., Lesieur M. and Brissaud A., 1974, A Markovian random coupling model for turbulence. J. Fluid Mech., Vol. 65, part 1, pp. 145-152.

Ferziger J.H., 1982, state of the art in subgrid scale modeling. Num.and Phys. Asp. Aerod. Flows, T. Cebeci, pp. 53-68. New-York : Springer 636. Kraichnan R.H., 1961, Dynamics of nonlinear stochastic systems. Journ. Math. Phys. Vol. 2, no ${ }^{\circ}$, pp. 124-148.

Kraichnan R.H., 1970, Convergents to turbulence functions. J. Fluid Mech., Vol. 41, part 1, pp. 189-217.

Kraichnan R.H., 1971, An almost-Markovian Galilean-invariant turbulence mode. J. Fluid Mech., Vol. 47, part 3, pp. 513-524.

Kraichnan R.H., 1976, Eddy viscosity in two and three dimensions. Journ. Atm. Sci., Vol. 33, pp. 1521-1536.

Leslie D.C. and Quarini G.I., 1979, The application of turbulence theory to the formulation of subgrid modelling procedures. J. Fluid Mech., Vol. 91, part 1, pp. 65-91.

Leith C.E., 1971, Atmospheric predictability and two-dimensional turbulence. Journ. Atm. Sci., Vol. 28, n० 2, pp. 145-16I.

orszag S.A., 1970, Analystical theories of turbulence. J. Fluid Mech., Vol. 41, part 2, pp. 363-386.

Orszag S.A. and Patterson G.S., 1972, Numerical simulation of threedimensional homogeneous isotropic turbulence. Phys. Rev. Letter.

Pouquet A., Lesieur M., André J.C. and Basdevant C., 1975, Evolution of high Reynolds number two-dimensional turbulence. J. Fluid Mech., Vol. 72, part 2, pp. 305-319.

Rogallo R.S., 1981, Numerical experiments in homogeneous turbulence. NASA Techn. Mem. $n^{\circ} 81315$, sept. 81 .

Rose H.A., 1977, Eddy diffusivity, Eddy noise and subgrid-scale modeling. Journal of Fluid Mech., Vol. 81, pp. 719-734. 